\title{
20 years' experience in the management of Zenker's diverticulum in a third-level hospital
}

\author{
J. Ruiz-Tovar, J. Pérez de Oteyza, M. V. Collado, R. Rojo and A. García-Villanueva \\ Department of General and Digestive Surgery. University Hospital "Ramón y Cajal”. Madrid, Spain
}

\begin{abstract}
Zenker's diverticulum arises in the posterior wall of the pharynx, above the cricopharyngeal muscle, secondary to a functional cricopharyngeal disorder. We describe our experience with the management of Zenker's diverticulum from 1985 to this day in a third-level hospital. We review clinical data from 27 patients $(78 \%$ males) with a mean age of 60.4 years. The most common clinical manifestations were dysphagia, regurgitation, syalorrhea, cough, and weight loss. All cases were diagnosed using an esophagogram. A diverticulectomy with cricopharingeal myotomy was performed in $74 \%$ of patients. Complications developed in 5 cases $(21 \%)$, and the recurrence rate was $4 \%$ ( 1 of 3 cases, where myotomy was not performed).
\end{abstract}

Key words: Zenker's diverticulum. Pharyngoesophageal diverticulum. Cricopharyngeal myotomy. Diverticulectomy. Diverticulopexy.

Ruiz-Tovar J. 20 years' experience in the management of Zenker's diverticulum in a third-level hospital. Rev Esp Enferm Dig 2006; 98: 429-435.

\section{INTRODUCTION}

Zenker's diverticulum was first described in 1769 by Ludlow (1). It is a sac-like deformation in the posterior wall of the pharynx that develops in the posterior mid line between the thyropharyngeal and cricopharyngeal muscles, above the level of the upper esophageal sphincter. It is currently accepted that its origin is a primary dysfunction of the cricopharyngeal muscle, which cannot re-

Recibido: 21-09-05.

Aceptado: 23-01-06.

Correspondencia: Jaime Ruiz-Tovar. Corazón de María, 64, 7º J. 28002 Madrid. e-mail: jruiztovar@gmail.com lax adequately and thus gives rise to a high pressure inside the pharynx when swallowing, and the formation of a secondary pulsion diverticulum. Its exact nature remains unclear to this day, with discrepancies between numerous studies (2).

Cricopharyngeal dysfunction and the presence of a sac condition the appearance of clinical manifestations. Most frequent symptoms are dysphagia, regurgitation and halitosis, although it sometimes manifests as a swallowing noise or the presence of a neck lump (3). The current treatment of symptomatic Zenker's diverticulum is surgery, approaching the diverticulum and cricopharyngeal muscle.

The aim of this study was to describe the acquired experience with the diagnosis and treatment of Zenker's diverticulum in a third-level hospital during a period of 20 years.

\section{MATERIAL AND METHODS}

A retrospective study of 27 patients diagnosed with Zenker's diverticulum was performed at the University Hospital "Ramón y Cajal" (Madrid, Spain) during the period between March 1985 and September 2005. Clinical data were obtained from a review of clinical records.

A descriptive statistical study was performed using the program SPSS 11.5 for Windows. Quantitative variables following a Gaussian model were defined by mean and range. For non-Gaussian variables median rather than mean values were used. Qualitative variables were defined by number of cases and percentage.

\section{Surgical technique}

After general anesthesia induction the patient is placed in the supine position with the neck rotated to the right. A 


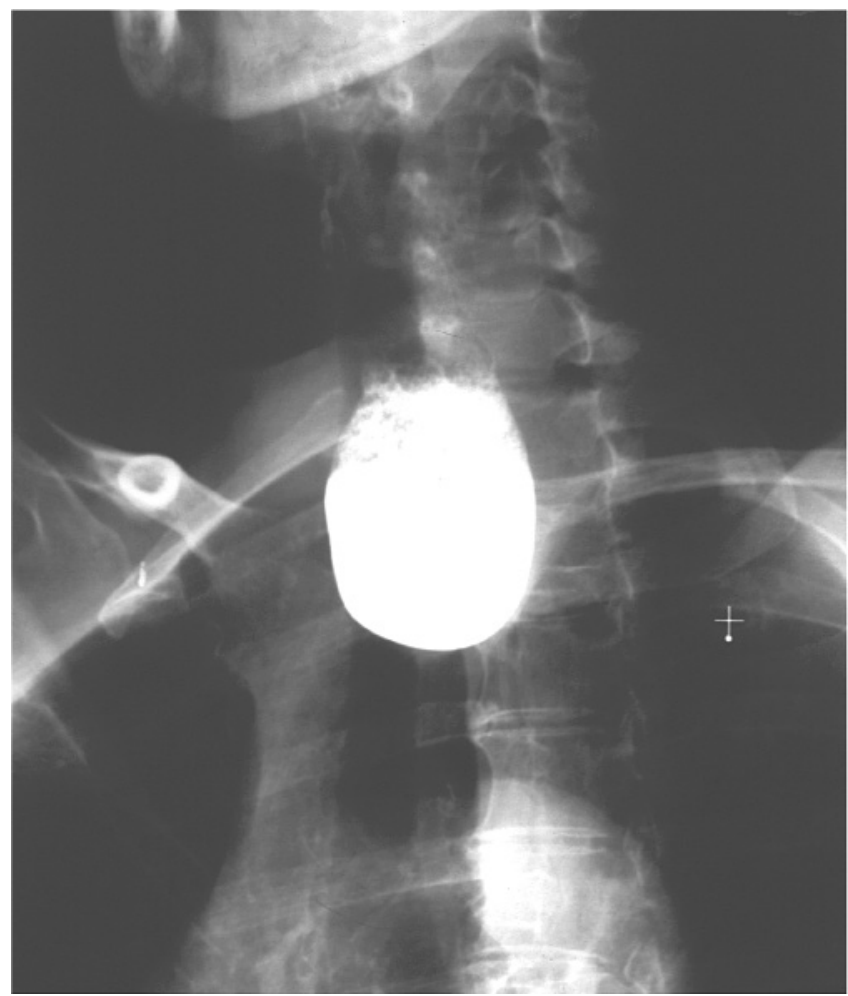

Fig. 1.- Zenker's diverticulum image (esophagogram). Imagen de divertículo de Zenker en esofagograma.

left cervicotomy is performed over the anterior border of the sternocleidomastoideus muscle, displacing it laterally. The anterior portion of the homohyoideus muscle is retracted without cutting it. The superior and medium thyroideal vessels are ligated and cut. One proceeds to the identification of the left laryngeal recurrent nerve, avoiding its damage. After surrounding the cervical esophagus, the diverticulum and its neck are dissected following a 2-4 cm myotomy of the cricopharyngeal muscle in most cases. Finally, the resection of diverticulum is performed, and the esophageal wall is mechanically closed using GIA or hand sewed. A drain is placed in the surgical bed and the incision is closed.

\section{RESULTS}

Clinical data are described in table I.

Dysphagia involved both solids and liquids. Regurgitation consisted of partially digested or non-digested food, increasing in the supine position. Asymptomatic cases were incidentally diagnosed when undergoing an esophagogram for another reason.

A hiatal hernia was seen in the esophagogram of $19 \%$ of cases.

Esophageal manometry showed a high-pressure upper esophageal sphincter in all 3 patients who underwent this
Table I

\begin{tabular}{|c|c|c|c|}
\hline Age & 60.4 years (range $43-91$ years) & & \\
\hline Gender & Male & 21 cases & $(78 \%)$ \\
\hline & Female & 6 cases & $(22 \%)$ \\
\hline Clinical manifestations & Regurgitation & 25 cases & $(93 \%)$ \\
\hline & Dysphagia & 24 cases & $(89 \%)$ \\
\hline & Syalorrhea & 6 cases & $(22 \%)$ \\
\hline & Weight loss & 6 cases & $(22 \%)$ \\
\hline & Cough & 6 cases & $22 \%)$ \\
\hline & Halitosis & 5 cases & $(19 \%)$ \\
\hline & Aspiration pneumonia & 5 cases & $(19 \%)$ \\
\hline & Dysphonia & 3 cases & $(11 \%)$ \\
\hline & Neck lump & 2 cases & $(7 \%)$ \\
\hline & Asymptomatic & 4 cases & $(15 \%)$ \\
\hline $\begin{array}{l}\text { Mean duration of symptoms } \\
\text { to diagnosis }\end{array}$ & 15 months (range 4-36 months) & & \\
\hline Laboratory studies & Esophagogram & 27 cases & $(100 \%)$ \\
\hline & Esophagoscopy & 9 cases & $(33 \%)$ \\
\hline & Esophageal manometry & 3 cases & $(11 \%)$ \\
\hline & Thorax CT-scan & 1 case & $(4 \%)$ \\
\hline Mean size & $2.9 \mathrm{~cm}$ (range 1.2-4.5 cm) & & \\
\hline Treatment & $\begin{array}{l}\text {-Diverticulectomy associated } \\
\text { with cricopharyngeal myotomy } \\
\text {-Diverticulectomy without }\end{array}$ & 20 cases & $(74 \%)$ \\
\hline & $\begin{array}{l}\text { myotomy } \\
\text {-Palliative gastrostomy }\end{array}$ & 3 cases & $(11 \%)$ \\
\hline & (non-surgical patients) & 1 case & $(4 \%)$ \\
\hline & $\begin{array}{l}\text {-Observation (asymptomatic } \\
\text { patients) }\end{array}$ & 3 cases & $(11 \%)$ \\
\hline Surgical time & 95 minutes (range 55-130 min) & & \\
\hline Median hospital stay & 6 days (range 3-23 days) & & \\
\hline Post-surgical recurrence & & 1 case & $(4 \%)$ \\
\hline Surgical complications & & 5 cases & $(21 \%)$ \\
\hline
\end{tabular}

study. In 2 patients an incomplete relaxation of the upper esophageal sphincter and pharynx-esophageal discoordination was also observed. A chest CT scan was performed in one case of aspirative pneumonia -it showed a dilated cervical esophagus suggesting Zenker's diverticulum, which was confirmed by an esophagogram.

Most cases began oral feeding 24-48 hours after surgery. Pathology did not reveal any case of carcinoma in association with Zenker's diverticulum.

Follow-up was performed for all operated patients 6 months after surgery. The only case of recurrence developed in one patient where a diverticulectomy without cricopharyngeal myotomy had been performed, who was again symptomatic 23 months after surgery. A new diverticulectomy, associated in this case with myotomy, was performed. Post-surgical complications included 1 case of upper esophageal stenosis 3 weeks after surgery, which was solved with endoscopic dilatations. One case presented with a transient recurrent nerve paralysis, which was treated with steroids. One patient developed a 
cervical abscess requiring surgical drain and antibiotics. An esophageal fistula developed in 2 subjects conservatively managed with parenteral feeding.

\section{DISCUSSION}

Zenker's diverticulum is an infrequent pathology in young people, being considered an acquired entity. Its usual development in advanced-age patients as described in the literature is consistent with our results (3). In spite of this, some authors have described a familiar aggregation (4). It is remarkable the predominance of this pathology among males in our series, which has already been described by other authors $(5,6)$.

Dysphagia, regurgitation and halitosis appeared as typical symptoms of Zenker's diverticulum. Weight loss could be attributed to dysphagia, avoiding the patient oral feeding in many cases (5). The content of the diverticulum, when aspirated, causes aspirative pneumonia. Peracchia et al. (6) described recurrent aspirative pneumonia in up to $20 \%$ of cases.

Zenker's diverticulum coexists often with hiatal hernia, a usual pathology in aged patients; Dodds (7) describe a $60 \%$ prevalence of gastroesophageal reflux and hiatal hernia in the general population over 60 . The concurrence of both morbid conditions can be more probably attributed to coexistence in the same age range than to an association between them with a common pathogenetic base.

Barium esophagogram is the most used technique for the diagnosis of esophageal diseases, being the first one to perform in Zenker's diverticulum. Esophagoscopy is the most direct method to establish the cause of mechanic dysphagia. Moreover, there is a risk of penetration and perforation of Zenker's diverticulum. This is the reason why it is considered as a second-choice technique, the previous performance of an esophagogram is advisable to help the endoscopist. Anyway, endoscopy is used as the first-choice technique in experienced centers. Manometry registers the pressure in different places of the esophagus and determines changes in the upper esophageal sphincter (8).

No carcinoma associated with a diverticulum appeared in any of our cases. Bradley et al. (9) describe the presence of carcinomas inside the diverticulum in $0.4 \%$ of cases, this being an infrequent condition that nonetheless needs that attention be paid to it.

In all 23 operated patients in our study (one patient underwent palliative gastrostomy), open diverticulectomy associated with cricopharyngeal myotomy was performed in most patients. Some authors have reported a lower recurrence rate when myotomy is associated with diverticulectomy (10), but there are very few comparative studies to confirm this hypothesis. Our experience reveals that the only patient who presented recurrence was one of the three cases undergoing diverticulectomy without myotomy. Lerut et al. (10) also described a higher complication rate when myotomy is not performed (21.7 vs. 11.3\%). In our series all complications appeared in cases where myotomy was associated, except for the patient who suffered from a transient recurrent nerve paralysis, who had undergone diverticulectomy alone. Konowitz and Biller (11) reported that diverticulopexy associated with cricopharyngeal myotomy is an alternative resulting in lower morbidity and shorter hospital stays when compared to diverticulectomy, with similar efficiency. The diverticulum is sewn to the retropharyngeal fascia or spinal anterior ligament in this approach. Aly et al. (3) recommend only myotomy for small diverticula under $1 \mathrm{~cm}$ in size, diverticulopexy associated with myotomy for moderate ones, and diverticulectomy associated with myotomy for big diverticula.

Recently, endoscopic techniques are gaining lots of adepts. In this approach the surgeon cuts with electrocoagulation or an endostapler the mucosa and muscle tissues of the wall, thus separating the diverticulum's lumen from the esophageal one. The benefits of this technique include: low morbidity, shorter time to oral feeding, shorter hospital stay and convalescence, shorter surgical time, similar results to open surgery, and lower costs (12-14); it is thus considered by some authors the treatment of choice for Zenker's diverticulum (15). Safden et al. (12) describe in a comparative study -endoscopic approach vs. open surgery- a significant reduction in hospital stay with the endoscopic approach (10 vs. 3.9 days), with oral feeding from the first day after surgery, and higher patient satisfaction; results were similar to those of open surgery in terms of efficiency, and complication and recurrence rates. Von Doersten and Byl (16) describe in a 40-case series satisfactory results in $90 \%$, with a $5-10 \%$ morbidity and complications rate. Most dangerous complications were bleeding, pneumomediastinum, and mediastinitis, although they were infrequent. Other minor complications were sore throat, lacerations of the oral mucosa, transient paralysis of vocal cords, and a foreign-body sensation in cases where the endostapler was used. Aly et al. (3) recommend to avoid the use of the endostapler for diverticula smaller than $2 \mathrm{~cm}$ and bigger than $10 \mathrm{~cm}$ in size, because of technical difficulties. In such cases where recurrence takes place, endoscopic reoperation is feasible with no increase in morbidity (17).

In conclusion, Zenker's diverticulum is a condition often resulting in mild symptoms, but interfering with quality of life; some times, however, it causes important manifestations (weight loss, nutritional deficiency secondary to dysphagia or aspirative pneumonia) that warrant treatment for all symptomatic diverticula. An esophagogram is the technique of choice for diagnosis, although endoscopy by experienced hands is a useful alternative. The most widely used treatment in our medium is diverticulectomy associated with myotomy, with recurrence being associated with lack of myotomy. 


\section{REFERENCES}

1. Ludlow A. A case of obstructed deglution from a preternatural dilatation of, and bag formed in, the pharynx. Med Observ Inq 1769; 3: 85101.

2. Cook IJ, Gabb M, Panagopoulos V, Jamieson GG, Dodds WJ, Dent J, et al. Pharyngeal (Zenker's) diverticulum is a disorder of upper esophageal sphincter opening. Gastroenterology 1992; 103: 1229-35.

3. Aly A, Devitt G, Jamieson GG. Evolution of surgical treatment for pharyngeal pouch. Br J Surg 2004; 91: 657-64.

4. Bjork H. Pathogenesis of hypopharyngeal diverticulum with special reference to heredity. Acta Otolaryngol 1952; 42: 202-7.

5. Mulder CJ, den Hartog G, Robijn RJ, Thies JE. Flexible endoscopic treatment of Zenker's diverticulum: a new approach. Endoscopy 1995; 27: 438-42.

6. Peracchia A, Bonavina L, Narne S, Segalin A, Antoniazzi L, Marotta G. Minimally invasive surgery for Zenker's diverticulum: analysis of results in 95 consecutive patients. Arch Surg 1998; 133: 695-700.

7. Dodds WJ. The pathogenesis of gastroesophageal reflux disease. AJR Am J Roentgenol 1988; 151: 49-56.

8. Rubesin SE. Oral and pharyngeal dysphagia. Gastroenterol Clin North Am 1995; 24: 331-52.

9. Bradley PJ, Kochaar A, Quraishi MS. Pharyngeal pouch carcinoma: real or imaginary risks? Ann Otol Rhinol Laryngol 1999; 108: 1027 32.
10. Lerut T, van Raemdonck D, Guelinckx P, Dom R, Geboes K. Zenker's diverticulum: is a myotomy of the cricopharyngeus useful? How long should it be? Hepatogastroenterology 1992; 39: 127-31.

11. Konowitz PM, Biller HF. Diverticulopexy and cricopharyngeal myotomy: treatment for the high-risk patient with a pharyngoesophageal (Zenker's) diverticulum. Otolaryngol Head Neck Surg 1989; 100: 146-53.

12. Safdar A, Curran A, Timon CV. Endoscopic stapling vs conventional methods of surgery for pharyngeal pouches: results, benefits and modifications. IR Med J 2004; 97: 75-6.

13. Scher RL, Richtsmeier WJ. Endoscopic staple-assisted esophagodiverticulostomy for Zenker's diverticulum. Laryngoscope 1996; 106: 951-6.

14. Richtsmeier WJ. Endoscopic management of Zenker diverticulum: The staple-assisted approach. Am J Medicine 2003; 115: 175S-178S.

15. Altman JI, Genden EM, Moche J. Fiberoptic endoscopic-assisted diverticulotomy: a novel technique for the management of Zenker's diverticulum. Ann Otol Rhinol Laryngol 2005; 114: 347-51.

16. Von Doersten PG, Byl FM. Endoscopic Zenker's diverticulum (Dohlman procedure): forty cases reviewed. Otolaryngol Head Neck Surg 1997; 116: 209-12.

17. Van Eeden S, Lloyd RV, Tranter RM. Comparison of the endoscopic stapling technique with more established procedures for pharyngeal pouches: results and patients satisfaction survey. J Laryngol Otol 1999; 113: 237-40. 